Article

\title{
Prognostic Value of Early Intermittent Electroencephalography in Patients after Extracorporeal Cardiopulmonary Resuscitation
}

\author{
Yong Oh Kim ${ }^{1}$, Ryoung-Eun Ko ${ }^{2}$, Chi Ryang Chung ${ }^{2}{ }^{\oplus}$, Jeong Hoon Yang ${ }^{2,3}$, Taek Kyu Park ${ }^{3}$, \\ Yang Hyun Cho ${ }^{4}$, Kiick Sung ${ }^{4}$, Gee Young Suh ${ }^{2,5}$ and Jeong-Am Ryu ${ }^{2,6, *(1)}$ \\ 1 Department of Emergency Medicine, Dankook University Hospital, Dankook University School of Medicine, \\ Cheonan 31116, Korea; ggggmmmmaaaail@gmail.com \\ 2 Department of Critical Care Medicine, Samsung Medical Center, \\ Sungkyunkwan University School of Medicine, Seoul 06351, Korea; ryoungeun.ko@samsung.com (R.-E.K.); \\ icu.chung@samsung.com (C.R.C.); jhysmc@gmail.com (J.H.Y.); suhgy@skku.edu (G.Y.S.) \\ 3 Division of Cardiology, Department of Medicine, Samsung Medical Center, \\ Sungkyunkwan University School of Medicine, Seoul 06351, Korea; taekkyu.park@samsung.com \\ 4 Department of Thoracic and Cardiovascular Surgery, Samsung Medical Center, \\ Sungkyunkwan University School of Medicine, Seoul 06351, Korea; yanghyun.cho@samsung.com (Y.H.C.); \\ kiick.sung@samsung.com (K.S.) \\ 5 Division of Pulmonary and Critical Care Medicine, Department of Medicine, Samsung Medical Center, \\ Sungkyunkwan University School of Medicine, Seoul 06351, Korea \\ 6 Department of Neurosurgery, Samsung Medical Center, Sungkyunkwan University School of Medicine, \\ Seoul 06351, Korea \\ * Correspondence: lamyud.ryu@samsung.com; Tel.: +82-2-3410-6399
}

Received: 18 May 2020; Accepted: 3 June 2020; Published: 4 June 2020

\begin{abstract}
The aim of this study was to investigate whether early intermittent electroencephalography (EEG) could be used to predict neurological prognosis of patients who underwent extracorporeal cardiopulmonary resuscitation (ECPR). This was a retrospective and observational study of adult patients who were evaluated by EEG scan within $96 \mathrm{~h}$ after ECPR. The primary endpoint was neurological status upon discharge from the hospital assessed with a Cerebral Performance Categories (CPC) scale. Among 69 adult cardiac arrest patients who underwent ECPR, 17 (24.6\%) patients had favorable neurological outcomes (CPC score of 1 or 2). Malignant EEG patterns were more common in patients with poor neurological outcomes (CPC score of 3,4 or 5 ) than in patients with favorable neurological outcomes $(73.1 \%$ vs. $5.9 \%, p<0.001)$. All patients with highly malignant EEG patterns $(43.5 \%)$ had poor neurological outcomes. In multivariable analysis, malignant EEG patterns and duration of cardiopulmonary resuscitation were significantly associated with poor neurological outcomes. In this study, malignant EEG patterns within $96 \mathrm{~h}$ after cardiac arrest were significantly associated with poor neurological outcomes. Therefore, an early intermittent EEG scan could be helpful for predicting neurological prognosis of post-cardiac arrest patients after ECPR.
\end{abstract}

Keywords: cardiopulmonary resuscitation; extracorporeal membrane oxygenation; neurological prognosis; electroencephalography

\section{Introduction}

Neurological prognosis is one of the most important issues in patients who survive a cardiac arrest $[1,2]$. It is important to estimate the reversibility of cerebral function in patients after return of spontaneous circulation. It may prevent inappropriate continuation of intensive treatment in 
patients who are predicted to have poor neurological outcomes [2,3]. Recently, extracorporeal membrane oxygenation (ECMO) has been increasingly used as an adjuvant therapy of conventional cardiopulmonary resuscitation (CPR), providing oxygenated blood and hemodynamic support in the absence of spontaneous cardiac circulation [4,5].

Autoregulation of cerebral blood flow may be changed in patients resuscitated from cardiac arrest [6]. It is difficult to predict how highly oxygenated continuous flow by ECMO affects cerebral autoregulation and neurological recovery in the setting of extracorporeal cardiopulmonary resuscitation (ECPR) [2]. In previous studies of ECPR, several predictors of mortality have been reported. However, limited data are available on neurological prognosis after ECPR [7].

Among electrophysiologic studies, electroencephalography (EEG) has been most widely used as one of the assessment tools for survivors after cardiac arrest [8]. In the setting of ECPR, whether an early intermittent EEG scan may be helpful for systemically estimating neurological outcomes of survivors has not been reported yet. Therefore, the objective of this study was to investigate whether an early intermittent EEG scan could be used to predict neurological outcomes of patients who underwent ECPR.

\section{Methods}

\subsection{Study Population and Design}

This was a retrospective, single-center, observational study of adult patients who underwent ECPR during hospitalization between February 2012 and December 2018. This study was approved by the Institutional Review Board of Samsung Medical Center (IRB no. SMC 2019-05-002). The requirement for informed consent was waived due to its retrospective nature. Clinical and laboratory data were collected by a trained study coordinator using a standardized case report form. Inclusion criteria were: (1) those who underwent ECPR during the study period; (2) those who had decreased mentalities (a score of $<13$ on the Glasgow Coma Scale) on EEG scan after cardiac arrest; and (3) those whose EEG scans were performed within $96 \mathrm{~h}$ after ECPR. Exclusion criteria were: (1) those who were under 18 years of age; (2) those with malignancy whose life expectancy was less than 1 year; (3) those with insufficient medical records; (4) those with causes of death verified to be other than brain death; and (5) those with a history of head trauma or a chronic neurological abnormality upon admission to the intensive care unit (ICU). Ultimately, a total of 69 patients with an EEG scan who were resuscitated by veno-arterial ECMO were analyzed in this study (Figure 1).

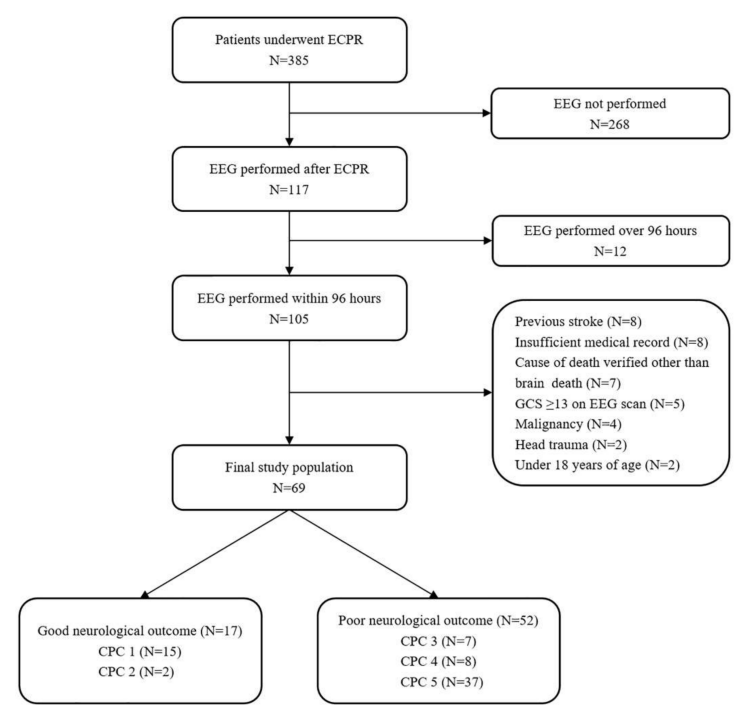

Figure 1. Study flow chart. ECPR, extracorporeal cardiopulmonary resuscitation; EEG, electroencephalography; GCS, Glasgow Coma Scale; CPC, Cerebral Performance Categories. 


\subsection{Endpoints and Definitions}

In this study, ECPR was defined as a successful veno-arterial ECMO implantation and pump-on with chest compression for external cardiac massage during index procedure in patients with cardiac arrest. When a return of spontaneous circulation occurs during ECMO cannulation, practitioners typically do not remove the cannula or stop the ECMO pump-on process [7]. Surface cooling and the degree of targeted temperature were determined by each intensivist in the ICU according to the targeted temperature management protocol [9]. The primary endpoint was the neurological status on discharge from the hospital. It was assessed with the Glasgow-Pittsburgh Cerebral Performance Categories (CPC) scale (scores range from 1 to 5) [10]. CPC scores of 1 and 2 were classified as favorable neurological outcomes while CPC scores of 3,4 and 5 were considered as poor neurological outcomes [11,12]. Medical records were thoroughly reviewed. Patients were graded on the CPC score by two independent neurologists. If the CPC scores did not match between the two neurologists, they discussed it and reached an agreement. A neurointensivist, attending physician or consultant neurologist determined EEG scan. An EEG scan was performed to identify causes of decreased consciousness or to predict neurological outcomes in ECPR patients. However, an EEG scan was not performed for patients who had a rapid recovery of mentality and neurological deficits. An EEG scan was also performed when patients had accompanied seizures or abnormal movements. If sedatives, analgesics or antiepileptic drugs were administrated to patients after ECPR, these drugs were not stopped during the EEG scan. EEG was performed using a 64-channel digital video-EEG system (Nicolet Biomedical, Inc., Madison, WI, USA). Surface electrodes were placed according to the international 10-20 system. Additional electrodes were placed whenever needed [13]. EEG patterns of ECPR patients were defined using the EEG terminology of the American Clinical Neurophysiology Society $[14,15]$. Malignant EEG patterns were defined as highly malignant EEG patterns and moderate malignant EEG patterns. Highly malignant EEG patterns were defined as suppressed background (amplitude $<10 \mu \mathrm{V}$, $100 \%$ of the recording) without discharges, suppressed background with superimposed continuous periodic discharges, or burst-suppression (periods of suppression with amplitude $<10 \mu \mathrm{V}$ constituting $>50 \%$ of the recording) with superimposed discharges or without discharges [15] (Figure 2).

A

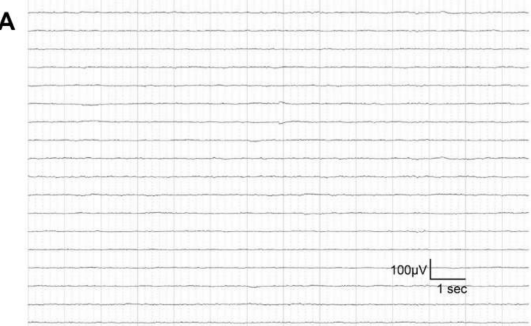

C

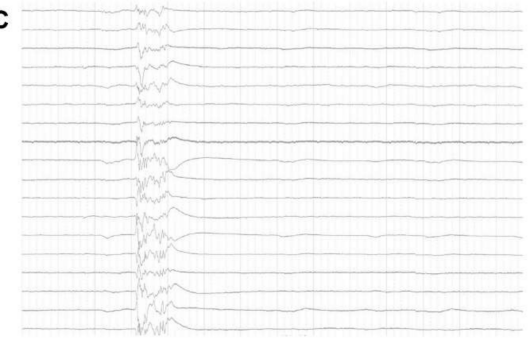

B

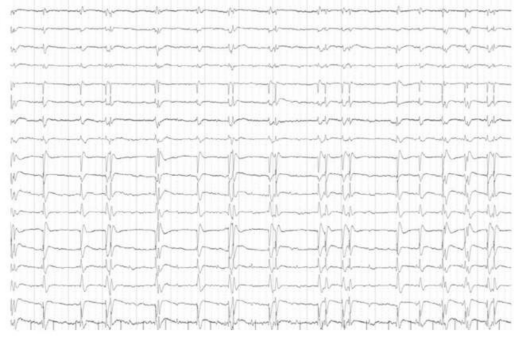

D

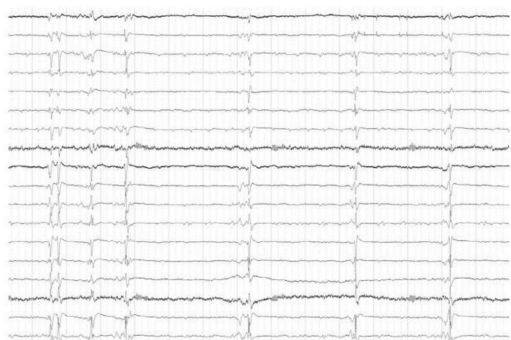

Figure 2. Highly malignant patterns of electroencephalography in patients after extracorporeal cardiopulmonary resuscitation. (A) Suppressed background without discharges; (B) Suppressed background with superimposed continuous periodic discharges; (C) burst-suppression without discharges; and (D) burst-suppression with superimposed discharges.

Moderate malignant EEG patterns were defined as malignant periodic or rhythmic patterns (abundant periodic discharges; abundant rhythmic spikes, polyspikes, sharp waves, spike-and-wave 
or sharp-and-slow wave; unequivocal electrographic seizure), malignant background (discontinuous background; low voltage background; reversed anterior-posterior gradient) or unreactive EEG (absence of background reactivity or only stimulus-induced discharges) [15]. Benign EEG patterns were defined as an absence of all malignant features stated above. EEG findings were confirmed by three EEG specialists.

\subsection{Procedure}

CPR was led by the CPR team of the hospital. All facts related to the CPR scene were recorded by bedside nurses according to Utstein-Style guidelines [16]. When CPR was performed for more than $10 \mathrm{~min}$ or in the event of unstable vital signs or recurrent cardiac arrest, the institutional rapid response team contacted the on-call ECMO team leader, who along with the CPR leader assessed the patient and made a decision about whether to institute ECPR. ECPR was performed when a witnessed arrest was confirmed, when the arrest persisted despite conventional CPR lasting for more than $10 \mathrm{~min}$, and when the event that caused the arrest was considered reversible [4]. Cases in which ECPR was deferred included those with a short life expectancy ( $<6$ months), terminal malignancy, an unwitnessed collapse, limited physical activity or CPR undertaken for more than $60 \mathrm{~min}$ at the time of initial contact. Age alone did not constitute a contraindication to ECPR [4].

The ECMO team consisted of cardiologists, cardiovascular surgeons, intensivists, special nurses and perfusionists. Either a Capiox Emergency Bypass System (Terumo, Tokyo, Japan) or a Prolonged Life Support System (Maquet Cardiopulmonary, Hirrlingen, Germany) was used in all cases. A crystalloid solution such as normal saline or balanced solution was used for priming. No patient had blood-primed ECMO. A percutaneous vascular approach was tried initially in all cases using the Seldinger technique. When percutaneous cannulation failed, surgical cutdown exposure was performed [4]. Femoral vessels were the most common sites of vascular access using 14 to 17 French arterial cannulas and 20 to 24 French venous cannulas [7]. Cardiac compression was stopped once ECMO pump-on was successful during CPR. Anticoagulation was accomplished by a bolus injection of unfractionated heparin, followed by continuous intravenous heparin infusion to maintain an activated clotting time between 150 and $180 \mathrm{~s}$. The initial number of revolutions per minute of the ECMO device was adjusted to achieve an ideal cardiac index greater than $2.2 \mathrm{~L} / \mathrm{min} / \mathrm{m}^{2}$ of body surface area, central mixed venous oxygen saturation above $70 \%$, and a mean arterial pressure above $65 \mathrm{~mm} \mathrm{Hg}$ [7]. Blood pressure was monitored continuously through an arterial catheter. An artery in the right arm was used for arterial blood gas analysis to estimate cerebral oxygenation. After ECMO, necessary steps were taken to treat the cause of the arrest, such as percutaneous coronary intervention, coronary artery bypass grafting, heart transplantation, non-coronary cardiopulmonary surgery or non-cardiopulmonary surgery [7].

\subsection{Statistical Analyses}

All data are presented as medians and interquartile ranges (IQRs, Q1 Q3) for continuous variables and as numbers (percentages) for categorical variables. Data were compared using the Mann-Whitney $U$ test for continuous variables and the Chi-squared test or Fisher's exact test for categorical variables. Variables with $p$ values less than 0.2 in univariate analyses and clinically relevant variables were subjected to a stepwise multiple logistic regression model to obtain statistically meaningful predictor variables. They were EEG groupings by its pattern, age, target temperature management, first monitored rhythm, CPR duration, Glasgow Coma Scale on EEG scan and use of sedative or analgesic. Due to small event rates, we took the caution of the general rule of 10 events per variable before any routine application of statistical methods and applied the Firth's correction. Adequacy of the prediction model was determined using the Hosmer-Lemeshow test, along with the areas under the curve (AUCs). The predictive performance of malignant EEG patterns assessed using the AUCs of the receiver operating characteristic (ROC) curves for sensitivity vs. 1-specificity. The AUCs were compared using the nonparametric approach published by DeLong et al. [17] for two correlated AUCs. All tests were two-sided and $p<0.05$ was considered statistically significant. All data were analyzed using IBM 
SPSS version 20 (IBM, Armonk, NY, USA) and R Statistical Software (version 3.6.3; R Foundation for Statistical Computing, Vienna, Austria).

\section{Results}

\subsection{Baseline Characteristics and Clinical Outcomes}

The median patient age was 56 (IQR: 47-70) years. Of 69 patients included in this study, 52 (75.4\%) were males. Hypertension (42.0\%) and diabetes mellitus (33.3\%) were the most common comorbidities among patients who underwent ECPR. Hypertension was more common in patients with poor neurological outcomes than in patients with favorable neurological outcomes $(50.0 \%$ vs. $17.6 \%$, $p=0.005)$. A cardiac cause of arrest was verified in $59(85.5 \%)$ patients. Acute coronary syndrome was the main cause of cardiac arrest in $26(44.1 \%)$ patients. Fourteen $(20.3 \%)$ patients had a history of ischemic heart disease. Forty-seven (68.1\%) patients experienced cardiac arrest in the hospital while $22(31.9 \%)$ patients suffered cardiac arrest in an out-of-hospital setting. Compared with the group with favorable neurological outcomes, the group with poor neurological outcomes had a longer CPR duration $(p=0.005)$. Baseline characteristics of ECPR patients are presented in Table 1.

Table 1. Baseline characteristics.

\begin{tabular}{|c|c|c|c|}
\hline & $\begin{array}{c}\text { Favorable Neurological } \\
\text { Outcome }(n=17)\end{array}$ & $\begin{array}{l}\text { Poor Neurological } \\
\text { Outcome }(n=52)\end{array}$ & $p$ Value \\
\hline Age (y)-median (IQR) & $51.0(36.0-73.0)$ & $57.5(50.0-69.5)$ & 0.460 \\
\hline Gender, male-no. of patients (\%) & $15(88.2)$ & $37(71.2)$ & 0.206 \\
\hline Body mass index $\left(\mathrm{kg} / \mathrm{m}^{2}\right)$-median (IQR) & $23.5(21.3-27.7)$ & $25.2(22.2-28.7)$ & 0.354 \\
\hline \multicolumn{4}{|l|}{ Medical history-no. of patients (\%) } \\
\hline Hypertension & $3(17.6)$ & $26(50.0)$ & 0.039 \\
\hline Diabetes mellitus & $3(17.6)$ & $20(38.5)$ & 0.199 \\
\hline Current smoker & $6(35.3)$ & $11(21.2)$ & 0.331 \\
\hline Previous myocardial infarction & $2(11.8)$ & $12(23.1)$ & 0.491 \\
\hline Malignancy & $1(5.9)$ & $11(21.2)$ & 0.269 \\
\hline Dyslipidemia & $2(11.8)$ & $10(19.2)$ & 0.716 \\
\hline Target temperature management-no. of patients (\%) & $9(52.9)$ & $21(40.4)$ & 0.532 \\
\hline Type of cardiac arrest-no. of patients (\%) & & & 0.581 \\
\hline Out of hospital cardiac arrest & $4(23.5)$ & $18(34.6)$ & \\
\hline In-hospital cardiac arrest & $13(76.5)$ & $34(65.4)$ & \\
\hline Bystander witnessed cardiac arrest-no. of patients (\%) & $17(100)$ & $50(96.2)$ & 0.999 \\
\hline Bystander performed CPR-no. of patients (\%) & $16(94.1)$ & $48(92.3)$ & 0.999 \\
\hline First monitored rhythm-no. of patients (\%) & & & 0.167 \\
\hline Asystole & $1(5.9)$ & $11(21.2)$ & \\
\hline Pulseless electrical activity & $6(35.3)$ & $23(44.2)$ & \\
\hline Shockable rhythm (VT or VF) & $10(58.8)$ & $18(34.6)$ & \\
\hline Defibrillation-no. of patients (\%) & $12(70.6)$ & $30(57.7)$ & 0.510 \\
\hline CPR duration (min)-median (IQR) & $19.0(8.0-28.0)$ & $31.0(21.0-40.5)$ & 0.005 \\
\hline Location of ECMO insertion-no. of patients (\%) & & & 0.177 \\
\hline Emergency room & $9(52.9)$ & $24(46.2)$ & \\
\hline Intensive care unit & $4(23.5)$ & $23(44.2)$ & \\
\hline Cath room & $4(23.5)$ & $4(7.7)$ & \\
\hline Operation room & $0(0)$ & $1(1.9)$ & \\
\hline Cardiac cause of arrest-no. of patients (\%) & & & 0.999 \\
\hline Ischemic & $7(41.2)$ & $19(45.2)$ & \\
\hline Non-ischemic & $10(58.8)$ & $23(54.8)$ & \\
\hline
\end{tabular}

IQR: interquartile range; CPR: cardiopulmonary resuscitation; VT: ventricular tachycardia; VF: ventricular fibrillation; ECMO: extracorporeal membrane oxygenation.

Among the 69 adult cardiac arrest patients who underwent ECPR, 32 (46.4\%) survived until discharge from the hospital. Of these 32 survivors, 17 (24.6\%) had favorable neurological outcomes (CPC score of 1 or 2). The entire distribution of CPC scores is shown in Figure 1.

\subsection{Relationship between EEG and Neurologic Outcomes}

Sedatives or analgesics were used in 41 (59.4\%) patients who underwent ECPR. These drugs were used more in patients with favorable neurological outcomes than in patients with poor neurological outcomes $(88.2 \%$ vs. $50.0 \%, p=0.012)$. There was no significant difference in the use of antiepileptic 
drugs between the two groups of patients $(p=0.999)$. Characteristics on the EEG scan are presented in Table 2.

Table 2. Characteristics on electroencephalography scan.

\begin{tabular}{|c|c|c|c|}
\hline & $\begin{array}{c}\text { Favorable Neurological } \\
\text { Outcome }(n=17)\end{array}$ & $\begin{array}{l}\text { Poor Neurological } \\
\text { Outcome }(n=52)\end{array}$ & $p$ Value \\
\hline Interval between ECPR and EEG scan-no. of patients (\%) & & & 0.671 \\
\hline $0-12 \mathrm{~h}$ & $2(11.8)$ & $10(19.2)$ & \\
\hline $12-24 \mathrm{~h}$ & $4(23.5)$ & $10(19.2)$ & \\
\hline $24-48 \mathrm{~h}$ & $5(29.4)$ & $20(38.5)$ & \\
\hline $48-96 \mathrm{~h}$ & $6(35.3)$ & $12(23.1)$ & \\
\hline Reasons of EEG scan no. of patients (\%) & & & 0.724 \\
\hline For neurological outcome prediction or decreased mentality & $7(41.2)$ & $26(50.0)$ & \\
\hline Seizure or abnormal movement & $10(58.8)$ & $26(50.0)$ & \\
\hline Pupil reflex-no. of patients (\%) & & & 0.199 \\
\hline Both prompt & $13(76.5)$ & 27 (51.9) & \\
\hline One or both sluggish & $2(11.8)$ & $8(15.4)$ & \\
\hline One or both fix & $2(11.8)$ & $17(32.7)$ & \\
\hline Glasgow Coma Scale on EEG scan & $7.0(3.0-9.0)$ & $3.0(3.0-7.0)$ & 0.012 \\
\hline Use of sedative or analgesic-no. of patients (\%) & $15(88.2)$ & $26(50.0)$ & 0.012 \\
\hline Bolus infusion & $4(23.5)$ & $10(19.2)$ & 0.734 \\
\hline Continuous infusion & $15(88.2)$ & $24(46.2)$ & 0.006 \\
\hline Remifentanil & $8(47.1)$ & $13(25.0)$ & \\
\hline Midazolam & $7(41.2)$ & $9(17.3)$ & \\
\hline Fentanyl & $6(35.3)$ & $5(9.6)$ & \\
\hline Propofol & $4(23.5)$ & $2(3.8)$ & \\
\hline Use of antiepileptic drug & $4(23.5)$ & $13(25.0)$ & 0.999 \\
\hline
\end{tabular}

ECPR: extracorporeal cardiopulmonary resuscitation; EEG: electroencephalography.

Malignant EEG patterns were more common in patients with poor neurological outcomes than in patients with favorable neurological outcomes $(73.1 \%$ vs. $5.9 \%, p<0.001$, Table 3$)$. All patients with highly malignant EEG patterns $(43.5 \%)$ had poor neurological outcomes. Moderately malignant EEG patterns were reported in eight $(11.6 \%)$ patients with poor neurological outcomes and in only one $(1.4 \%)$ patient with a favorable neurological outcome. Regardless of the interval between ECPR and EEG scans, most patients with malignant EEG patterns had poor neurological outcomes in this study. In addition, all patients with myoclonic status epilepticus had poor neurological outcomes. Benign EEG patterns were more common in patients with favorable neurological outcomes than in patients with poor neurological outcomes $(94.1 \%$ vs. $26.9 \%, p<0.001$, Table 3$)$.

Table 3. Findings of electroencephalography.

\begin{tabular}{|c|c|c|c|}
\hline & $\begin{array}{l}\text { Favorable Neurological } \\
\text { Outcome }(n=17)\end{array}$ & $\begin{array}{l}\text { Poor Neurological } \\
\text { Outcome }(n=52)\end{array}$ & $p$ Value \\
\hline EEG findings-no. of patients (\%) & & & $<0.001$ \\
\hline Benign EEG & $16(94.1)$ & $14(26.9)$ & \\
\hline Malignant EEG & $1(5.9)$ & $38(73.1)$ & \\
\hline \multicolumn{4}{|l|}{ Highly malignant EEG } \\
\hline Suppressed background without discharges & $0(0)$ & $18(34.6)$ & \\
\hline $\begin{array}{l}\text { Suppressed background with continuous periodic } \\
\text { discharges }\end{array}$ & $0(0)$ & $2(3.8)$ & \\
\hline $\begin{array}{l}\text { Burst-suppression background with or without } \\
\text { discharges }\end{array}$ & $0(0)$ & $10(19.2)$ & \\
\hline \multicolumn{4}{|l|}{ Moderately malignant EEG } \\
\hline Malignant periodic or rhythmic patterns & $0(0)$ & $6(11.5)$ & \\
\hline Malignant background & $0(0)$ & $2(3.8)$ & \\
\hline Unreactive EEG & $1(5.9)$ & $0(0)$ & \\
\hline \multicolumn{4}{|l|}{ EEG patterns according to time interval-no. of patients (\%) } \\
\hline EEG performed within $24 \mathrm{~h}$ after ECPR & & & 0.001 \\
\hline Benign EEG patterns & $6(35.3)$ & $4(7.7)$ & \\
\hline Malignant EEG patterns & $0(0)$ & $16(30.8)$ & \\
\hline EEG performed over $24 \mathrm{~h}$ after ECPR & & & 0.001 \\
\hline Benign EEG patterns & $10(58.8)$ & $10(19.2)$ & \\
\hline Malignant EEG patterns & $1(5.9)$ & $22(42.3)$ & \\
\hline Accompanied clinical seizure-no. of patients (\%) & & & 0.121 \\
\hline Absence of clinical seizure & $7(41.2)$ & $28(53.8)$ & \\
\hline Sporadic seizure or myoclonus & $10(58.8)$ & $18(34.6)$ & \\
\hline Myoclonic status epilepticus & $0(0)$ & $6(11.5)$ & \\
\hline
\end{tabular}

ECPR: extracorporeal cardiopulmonary resuscitation; EEG: electroencephalography. 
In multivariable analysis, the only significant indicators were EEG grouping by its pattern and CPR duration. That is, malignant EEG patterns (adjusted odd ratio (OR): 36.43, 95\% confidence interval (CI): 4.632-1013.711) and CPR duration (adjusted OR: 1.06 per minute increase, 95\% CI: 1.00-1.138) were significantly associated with poor neurological outcomes in patients who underwent ECPR(Hosmer-Lemeshow Chi-squared $=2.32, d f=8, p=0.969)$ with the AUCs of $0.946(95 \%$ CI 0.893-0.999) (Table 4).

Table 4. Multivariable logistic regression of clinically relevant variables associated with poor neurological outcomes.

\begin{tabular}{lcc}
\hline & Adjusted OR (95\% CI) & $p$ Value \\
\hline Malignant EEG patterns & $36.43(4.632-1013.711)$ & $<0.001$ \\
First monitored rhythm & 1 & Reference \\
$\quad$ Asystole & $0.84(0.046-11.674)$ & 0.894 \\
$\quad$ Pulseless electrical activity & $0.19(0.011-1.622)$ & 0.135 \\
$\quad$ Shockable rhythm (VT or VF) & $1.06(1.009-1.138)$ & 0.020 \\
CPR duration (min) & $0.26(0.023-1.845)$ & 0.188 \\
Use of sedative or analgesic & & \\
\hline
\end{tabular}

OR: odd ratio; CI: confidence interval; EEG: electroencephalography; VT: ventricular tachycardia; VF: ventricular fibrillation; CPR: cardiopulmonary resuscitation. Variables with $p$ values less than 0.2 in univariate analyses and clinically relevant variables were subjected to a stepwise multiple logistic regression model to obtain statistically meaningful predictor variables. They were EEG groupings by its pattern, age, target temperature management, first monitored rhythm, CPR duration, Glasgow Coma Scale on EEG scan, and use of sedative or analgesic. To alleviate the small sample issue, we applied the Firth's correction.

Although there were no differences between the AUCs of malignant EEG patterns and CPR duration, the performance of a composite of these marker was strongly associated with poor neurological outcomes compared with the use of either marker alone ( $p=0.008$ and $p=0.006$, respectively) (Figure 3).

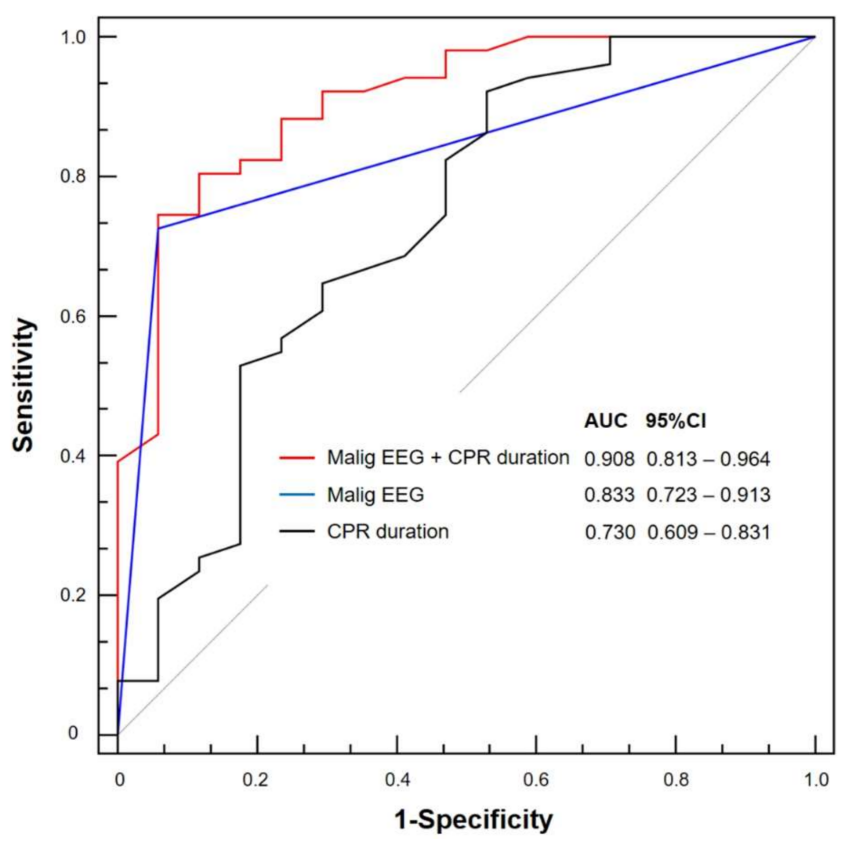

Figure 3. Receiver operating characteristic curves for predicting poor outcomes using malignant patterns of electroencephalography (EEG) and cardiopulmonary resuscitation (CPR) duration. Although there were no differences between the areas under the curve (AUCs) of malignant EEG patterns and CPR duration, the performance of a composite of these marker was strongly associated with poor neurological outcomes compared with the use of either marker alone ( $p=0.008$ and $p=0.006$, respectively). CI, confidence interval; Malig EEG, malignant EEG patterns. 


\section{Discussion}

In this study, we investigated whether intermittent EEG could be used to predict neurological outcomes of patients who underwent ECPR. The major findings of this study were as follows. First, regardless of sedation, malignant EEG patterns were more common in patients with poor neurological outcomes than in patients with favorable neurological outcomes. Especially, all patients with highly malignant EEG patterns had poor neurological outcomes. In addition, patients with moderate malignant EEG patterns had poor neurological outcome except for one patient. Second, benign EEG patterns alone did not necessarily imply a favorable neurological outcome. Third, in multivariable analysis, malignant EEG patterns and CPR duration were significantly associated with poor neurological outcomes in patients who underwent ECPR. Therefore, early intermittent EEG scans and CPR duration could be helpful for predicting neurological outcomes of post-cardiac arrest patients after ECPR.

EEG signals mainly reflect cerebral cortical function and some subcortical function [8]. EEG is very sensitive to ischemia because cortical neurons of the brain need a consistent blood supply to maintain signaling and integrity [8]. Therefore, the EEG scan is a standard and useful tool to predict neurological outcomes after cardiac arrest [3,15]. Especially, malignant EEG patterns such as suppressed background, status epilepticus, burst suppression, periodic patterns and unreactive EEG are associated with poor neurological prognosis after cardiac arrest $[8,15,18]$. In addition, early continuous waves with normal voltage could be a predictor of favorable neurological outcomes after cardiac arrest [19].

Cerebral autoregulation may be changed in survivors after cardiac arrest [6]. Highly oxygenated continuous ECMO flow could affect cerebral autoregulation after ECPR [2]. In addition, neurological outcomes may be affected by functional recovery of native heart and lung, the amount of ECMO support and changed cerebral autoregulation [2]. Altered cerebral hemodynamics by ECMO support may influence neurological outcomes after ECPR. Therefore, it is difficult to predict neurological prognosis by these changed situations after ECPR [2]. Ultimately, the interaction between cerebral autoregulation and ECMO flow may affect neurological recovery and prognosis in ECPR patients through mechanisms of primary ischemic damage and secondary additive injury [2]. Thus, EEG change by this interaction should be studied for neurological prediction after ECPR. However, there has been no report of EEG according to neurological outcomes after ECPR.

Sedation may confuse outcome predictions in survivors of cardiac arrests $[1,8,20]$. Sedatives are commonly used in survivors after cardiac arrest for $72 \mathrm{~h}$ as important confounders [1,20]. A motor response to noxious stimuli, corneal reflex, caloric testing and some electrophysiologic studies may also be confounded by sedation $[20,21]$. Although mild to moderate hypothermia does not significantly affect EEG in patients with induced hypothermia [8,22], a confounder accompanied by induced hypothermia such as analgesics, sedatives or artifacts from shivering, mechanical ventilator or electrical devices may affect the reliability of EEG interpretations [8]. However, a recent study has reported that the predictive performance of EEG after cardiac arrest is similar between patients with ongoing sedation and those without ongoing sedation [8,15]. In this study, sedation or targeted temperature management did not significantly affect the prediction of poor neurological outcomes after ECPR. Regardless of sedation or targeted temperature management, patients with malignant EEG patterns had poor neurological outcomes in this study.

Benign EEG patterns may be associated with a favorable neurological outcome in survivors after cardiac arrest [19]. Especially, early continuous waves with normal voltage could be a predictor of favorable neurological outcomes after cardiac arrest $[8,15,19]$. However, in previous studies, benign EEG patterns are not always associated with a good neurological outcome $[15,18]$. Additive secondary injury is characterized by an imbalance in post-resuscitation cerebral oxygen delivery and use [23]. This injury is associated with reperfusion injury, impaired autoregulation, fluctuations in oxygen support and arterial carbon dioxide, hyperthermia and concomitant anemia [23]. Early EEG findings may not be shown to be malignant EEG patterns in patients with poor neurological outcomes if the secondary cerebral injury is more serious than the primary cerebral injury. In this study, benign EEG patterns were not always associated with a favorable neurological outcome. In addition, intermittent 
EEG scans may be less sensitive for predicting favorable neurological outcomes than continuous EEG monitoring in this study.

It is not easy to predict neurological prognosis of survivors after cardiac arrest [24,25]. In addition, there is no optimal timing to assess prognosis after cardiac arrest [25]. Although highly malignant EEG patterns may be associated with poor prognosis, other EEG patterns may be ambiguous in predicting outcomes in various conditions after cardiac arrest $[15,26]$. Therefore, it may be unreasonable to predict the prognosis of survivors after ECPR based on EEG patterns alone. Early EEG scans may help to predict neurological outcomes and reduce uncertainty over coma prognostication after cardiac arrest [26]. Therefore, EEG should be used as one of the multimodal tools rather than an absolute tool for predicting prognosis. Eventually, a multimodal approach, including neurological examination, biomarker, brain imaging, evoked potential and EEG may be needed to predict neurological outcomes in patients after ECPR $[24,25]$.

This study has several limitations. First, this was a retrospective review. Thus, CPC score was determined based on medical records. By using two independent specialists' agreement on the score, any bias may be mitigated to some extent. In addition, although the cause of death had to be accurately verified, its identification was insufficient due to the retrospective nature of this study. Second, the nonrandomized nature of registry data might have resulted in selection bias. EEG scans were not protocol-based in their performance. Particularly, during the study period, EEG scans were not performed in all patients. They were only performed in patients with abnormal consciousness, seizure, abnormal movements or other symptoms. Although EEG scans were performed within $96 \mathrm{~h}$ following ECPR, a major limitation of this study might be that EEG scans were performed in different time settings. Lastly, our study has limited statistical power due to its small sample size. To alleviate the small sample issue, we applied the Firth's correction, which dampens the results somewhat. Although it still provides a valuable insight, prospective large-scale studies are needed to confirm the usefulness of early intermittent EEG scan in predicting neurological outcomes of patients after ECPR to arrive at evidence-based conclusions.

\section{Conclusions}

In this study, malignant EEG patterns within $96 \mathrm{~h}$ after cardiac arrest were significantly associated with poor neurological outcomes in patients who underwent ECPR. A multimodal approach is needed to predict neurological outcomes in patients after ECPR. Especially, an early EEG scan may help to predict neurological outcomes and reduce uncertainty about coma prognostication after ECPR.

Author Contributions: Conceptualization, Y.O.K. and J.A.R.; methodology, Y.O.K. and J.A.R.; resources, Y.O.K., R.-E.K. and J.A.R.; supervision, C.R.C., J.H.Y., T.K.P., Y.H.C., K.S., G.Y.S. and J.-A.R.; writing-original draft preparation, Y.O.K. and J.A.R. All authors have read and agreed to the published version of the manuscript.

Funding: This research received no external funding.

Acknowledgments: We would like to thank the excellent statistical support of Keumhee C. Carriere, and Joonghyun Ahn, of the Samsung Biomedical Research Institute. We also would like to thank the nursing director of the neurosurgical intensive care unit, Hye Jung Kim, who provided excellent advice and fruitful discussions. We also thank all nurses of the neurosurgical intensive care unit at Samsung Medical Center.

Conflicts of Interest: The authors declare no competing interests.

Availability of Data and Materials: Regarding data availability, our data are available on the Harvard Dataverse Network (http://dx.doi.org/10.7910/DVN/KYJNVA).

Consent for Publication: Not applicable. This study does not contain individual or personal data in any form (including individual details, images, or videos).

\section{References}

1. Ryu, J.A.; Chung, C.R.; Cho, Y.H.; Sung, K.; Suh, G.Y.; Park, T.K.; Song, Y.B.; Hahn, J.Y.; Choi, J.H.; Gwon, H.C.; et al. The association of findings on brain computed tomography with neurologic outcomes following extracorporeal cardiopulmonary resuscitation. Crit. Care 2017, 21, 15. [CrossRef] 
2. Ryu, J.A.; Chung, C.R.; Cho, Y.H.; Sung, K.; Jeon, K.; Suh, G.Y.; Park, T.K.; Lee, J.M.; Song, Y.B.; Hahn, J.Y.; et al. Neurologic Outcomes in Patients Who Undergo Extracorporeal Cardiopulmonary Resuscitation. Ann. Thorac. Surg. 2019, 108, 749-755. [CrossRef]

3. Sondag, L.; Ruijter, B.J.; Tjepkema-Cloostermans, M.C.; Beishuizen, A.; Bosch, F.H.; van Til, J.A.; van Putten, M.; Hofmeijer, J. Early EEG for outcome prediction of postanoxic coma: Prospective cohort study with cost-minimization analysis. Crit. Care 2017, 21, 111. [CrossRef]

4. Ryu, J.A.; Cho, Y.H.; Sung, K.; Choi, S.H.; Yang, J.H.; Choi, J.H.; Lee, D.S.; Yang, J.H. Predictors of neurological outcomes after successful extracorporeal cardiopulmonary resuscitation. BMC Anesthesiol. 2015, 15, 26. [CrossRef]

5. Ahn, C.; Kim, W.; Cho, Y.; Choi, K.S.; Jang, B.H.; Lim, T.H. Efficacy of extracorporeal cardiopulmonary resuscitation compared to conventional cardiopulmonary resuscitation for adult cardiac arrest patients: A systematic review and meta-analysis. Sci. Rep. 2016, 6, 34208. [CrossRef]

6. Sundgreen, C.; Larsen, F.S.; Herzog, T.M.; Knudsen, G.M.; Boesgaard, S.; Aldershvile, J. Autoregulation of cerebral blood flow in patients resuscitated from cardiac arrest. Stroke 2001, 32, 128-132. [CrossRef]

7. Park, S.B.; Yang, J.H.; Park, T.K.; Cho, Y.H.; Sung, K.; Chung, C.R.; Park, C.M.; Jeon, K.; Song, Y.B.; Hahn, J.Y.; et al. Developing a risk prediction model for survival to discharge in cardiac arrest patients who undergo extracorporeal membrane oxygenation. Int. J. Cardiol. 2014, 177, 1031-1035. [CrossRef]

8. Westhall, E. Electroencephalography as a Prognostic Tool after Cardiac Arrest. Semin. Neurol. 2017, 37, 48-59. [CrossRef]

9. Kang, M.J.; Lee, T.R.; Shin, T.G.; Sim, M.S.; Jo, I.J.; Song, K.J.; Jeong, Y.K. Survival and neurologic outcomes of out-of-hospital cardiac arrest patients who were transferred after return of spontaneous circulation for integrated post-cardiac arrest syndrome care: The another feasibility of the cardiac arrest center. J. Korean Med. Sci. 2014, 29, 1301-1307. [CrossRef]

10. Cummins, R.O.; Chamberlain, D.A.; Abramson, N.S.; Allen, M.; Baskett, P.J.; Becker, L.; Bossaert, L.; Delooz, H.H.; Dick, W.F.; Eisenberg, M.S.; et al. Recommended guidelines for uniform reporting of data from out-of-hospital cardiac arrest: The Utstein Style. A statement for health professionals from a task force of the American Heart Association, the European Resuscitation Council, the Heart and Stroke Foundation of Canada, and the Australian Resuscitation Council. Circulation 1991, 84, 960-975.

11. The Hypothermia after Cardiac Arrest Study Group. Mild therapeutic hypothermia to improve the neurologic outcome after cardiac arrest. N. Engl. J. Med. 2002, 346, 549-556. [CrossRef]

12. Rittenberger, J.C.; Raina, K.; Holm, M.B.; Kim, Y.J.; Callaway, C.W. Association between Cerebral Performance Category, Modified Rankin Scale, and discharge disposition after cardiac arrest. Resuscitation 2011, 82, 1036-1040. [CrossRef]

13. Kim, J.; Jung, D.S.; Hwang, K.J.; Seo, J.H.; Na, G.Y.; Hong, S.B.; Joo, E.Y.; Seo, D.W. Can an exercise bicycle be safely used in the epilepsy monitoring unit?: An exercise method to provoke epileptic seizures and the related safety issues. Epilepsy Behav. 2015, 46, 79-83. [CrossRef]

14. Hirsch, L.J.; LaRoche, S.M.; Gaspard, N.; Gerard, E.; Svoronos, A.; Herman, S.T.; Mani, R.; Arif, H.; Jette, N.; Minazad, Y.; et al. American Clinical Neurophysiology Society's Standardized Critical Care EEG Terminology: 2012 version. J. Clin. Neurophysiol. 2013, 30, 1-27. [CrossRef]

15. Westhall, E.; Rossetti, A.O.; van Rootselaar, A.F.; Wesenberg Kjaer, T.; Horn, J.; Ullen, S.; Friberg, H.; Nielsen, N.; Rosen, I.; Aneman, A.; et al. Standardized EEG interpretation accurately predicts prognosis after cardiac arrest. Neurology 2016, 86, 1482-1490. [CrossRef]

16. Cummins, R.O.; Chamberlain, D.; Hazinski, M.F.; Nadkarni, V.; Kloeck, W.; Kramer, E.; Becker, L.; Robertson, C.; Koster, R.; Zaritsky, A.; et al. Recommended guidelines for reviewing, reporting, and conducting research on in-hospital resuscitation: The in-hospital 'Utstein style'. American Heart Association. Circulation 1997, 95, 2213-2239. [CrossRef]

17. DeLong, E.R.; DeLong, D.M.; Clarke-Pearson, D.L. Comparing the areas under two or more correlated receiver operating characteristic curves: A nonparametric approach. Biometrics 1988, 44, 837-845. [CrossRef]

18. Amorim, E.; Rittenberger, J.C.; Baldwin, M.E.; Callaway, C.W.; Popescu, A. Malignant EEG patterns in cardiac arrest patients treated with targeted temperature management who survive to hospital discharge. Resuscitation 2015, 90, 127-132. [CrossRef] 
19. Oh, S.H.; Park, K.N.; Shon, Y.M.; Kim, Y.M.; Kim, H.J.; Youn, C.S.; Kim, S.H.; Choi, S.P.; Kim, S.C. Continuous Amplitude-Integrated Electroencephalographic Monitoring Is a Useful Prognostic Tool for Hypothermia-Treated Cardiac Arrest Patients. Circulation 2015, 132, 1094-1103. [CrossRef]

20. Samaniego, E.A.; Mlynash, M.; Caulfield, A.F.; Eyngorn, I.; Wijman, C.A. Sedation confounds outcome prediction in cardiac arrest survivors treated with hypothermia. Neurocrit. Care 2011, 15, 113-119. [CrossRef]

21. Young, G.B. Clinical practice. Neurologic prognosis after cardiac arrest. N. Engl. J. Med. 2009, 361, 605-611. [CrossRef] [PubMed]

22. Stecker, M.M.; Cheung, A.T.; Pochettino, A.; Kent, G.P.; Patterson, T.; Weiss, S.J.; Bavaria, J.E. Deep hypothermic circulatory arrest: I. Effects of cooling on electroencephalogram and evoked potentials. Ann. Thorac. Surg. 2001, 71, 14-21. [CrossRef]

23. Sekhon, M.S.; Ainslie, P.N.; Griesdale, D.E. Clinical pathophysiology of hypoxic ischemic brain injury after cardiac arrest: A “two-hit" model. Crit. Care 2017, 21, 90. [CrossRef] [PubMed]

24. Sandroni, C.; Cariou, A.; Cavallaro, F.; Cronberg, T.; Friberg, H.; Hoedemaekers, C.; Horn, J.; Nolan, J.P.; Rossetti, A.O.; Soar, J. Prognostication in comatose survivors of cardiac arrest: An advisory statement from the European Resuscitation Council and the European Society of Intensive Care Medicine. Resuscitation 2014, 85, 1779-1789. [CrossRef]

25. Taccone, F.; Cronberg, T.; Friberg, H.; Greer, D.; Horn, J.; Oddo, M.; Scolletta, S.; Vincent, J.L. How to assess prognosis after cardiac arrest and therapeutic hypothermia. Crit Care 2014, 18, 202. [CrossRef] [PubMed]

26. Bongiovanni, F.; Romagnosi, F.; Barbella, G.; Di Rocco, A.; Rossetti, A.O.; Taccone, F.S.; Sandroni, C.; Oddo, M. Standardized EEG analysis to reduce the uncertainty of outcome prognostication after cardiac arrest. Intensive Care Med. 2020, 46, 963-972. [CrossRef] [PubMed]

(C) 2020 by the authors. Licensee MDPI, Basel, Switzerland. This article is an open access article distributed under the terms and conditions of the Creative Commons Attribution (CC BY) license (http://creativecommons.org/licenses/by/4.0/). 